\title{
ERRATUM
}

\section{Broadcasting in Multi-Radio Multi-Channel and Multi-Hop Wireless Networks}

\author{
Li Li, Bin Qin, and Chunyuan Zhang
}

School of Computer Science

National University of Defense Technology

Changsha, Hunan province, 410073, China

liligfkd@163.com, qbsnatom.com, cyzhang@163.com

D. Krishnaswamy, T. Pfeifer, and D. Raz (Eds.): MMNS 2007, LNCS 4787, pp. 101-112, 2007.

(C) IFIP International Federation for Information Processing 2007

\section{DOI 10.1007/978-3-540-75869-3_9}

Due to copyright violation, this publication is no more valid. We sincerely apologize for any confusion and inconvenience. 